\title{
Suppression of visibility in a two-electron Mach-Zehnder interferometer
}

\author{
Ya. M. Blanter ${ }^{1,2}$ and Yuval Gefen ${ }^{2}$ \\ ${ }^{1}$ Kavli Institute of Nanoscience, Delft University of Technology, Lorentzweg 1, 2628 CJ Delft, The Netherlands \\ ${ }^{2}$ Department of Condensed Matter Physics, The Weizmann Institute of Science, Rehovot 76100, Israel
}

(Received 15 October 2007; published 20 December 2007)

\begin{abstract}
We investigate the suppression of the visibility of Aharonov-Bohm oscillations in a two-electron MachZehnder interferometer that leaves the single-electron current unchanged. In the case when the sources emit either spin-polarized or entangled electrons, partial distinguishability of electrons (coming from two different sources) suppresses the visibility. Two-particle entanglement may produce behavior similar to "dephasing" of two-particle interferometry.
\end{abstract}

DOI: 10.1103/PhysRevB.76.235319

PACS number(s): 73.23.Ad, 73.43.-f, 73.90.+f

\section{INTRODUCTION}

Aharonov-Bohm $(\mathrm{AB})$ interferometry is a centerpiece in studies of nanoscopic electronic systems. Of special interest are Mach-Zehnder interferometers (MZIs): ${ }^{1}$ The absence of backscattering gives rise to a large interference signal (large visibility) and allows measurements in strong magnetic fields.

One conceptual step beyond single-particle interferometry is a "two-particle interferometry," tailored after the Hanbury Brown-Twiss experiment. In particular, one can utilize a two-electron MZI which features two current sources and two detectors (Fig. 1). Such a device, suitable for the measurement of current cross correlations in the detectors, has been originally proposed by Samuelson-SukhorukovBüttiker (SSB). In Ref. 2, it was shown that while the current (and the noise) at each particular detector is insensitive to the $\mathrm{AB}$ flux through the interferometer, the current cross correlations between the two detectors do show $\mathrm{AB}$ oscillations. The latter are a direct consequence of particle indistinguishability: Measuring current signals at points $\mathrm{c}$ and $\mathrm{d}$ [Fig. 1(a)] may be due to an electron from a (b) absorbed at c (d) or at $\mathrm{d}$ (c). The product of the amplitudes of these two processes is flux sensitive. Had the two particles been distinguishable, it would have been possible to conclude, for example, that the electron detected at $\mathrm{c}(\mathrm{d})$ originated from a (b). Only one of the above amplitudes survives, hence flux insensitivity.

Trying to design an actual measurement of a two-electron interferometry, it is important to understand which manipulations can render the electrons distinguishable, in practice, suppress the AB signal. Evidently, one can suppress the interference by introducing dephasers at the interferometer's arms. However, this will also suppress single-particle interference. We do not discuss this option in this paper. ${ }^{3}$ Below, we focus on two different interference-suppressing scenarios. First, one can introduce a spin polarization of the sources. We show below that for opposite spin polarizations of the two sources, AB oscillations are suppressed, since electrons with opposite spin projections are distinguishable. The second scenario we consider is the entanglement of electrons in one of the sources. Intuitively, sources emitting states entangled with respect to electron spin might behave as "a little bit spin polarized," and thus induce a suppression of twoparticle interference.
In this paper, we calculate how the visibility in these two scenarios is reduced. In particular, our analysis reveals indeed that entangled electrons vs nonentangled electrons act as (partially) distinguishable particles.

Whereas spin-polarized electron sources can be considered within the existing scattering approach to electron transport, sources of entangled electrons present considerable difficulties. First, up to now, entanglement of electrons in solid state has never been demonstrated experimentally. There is a number of theoretical proposals (see Ref. 4 for recent review); most of them use interaction to create entangled pairs. For noninteracting electrons, the proposals include spatial separation of spin-polarized particles ${ }^{5,6}$ or, indeed, edge states in the quantum Hall effect regime. ${ }^{2,7}$ We also note that proposals for manifestations of non-Abelian statistics ${ }^{8,9}$ rely, in fact, on entanglement of the appropriate quasiparticles. A proper way to consider transport of such entangled pairs would be the development of full quantum-mechanical theory including the interaction effects. It is a major challenge, and it is unlikely that such theory will be developed anytime soon. Here, we follow a different avenue. We develop a transport theory for two particles along the same line as single-particle transport. We show that within this ap-

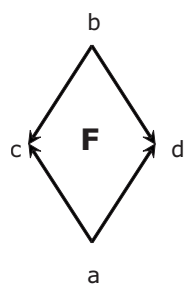

(a)

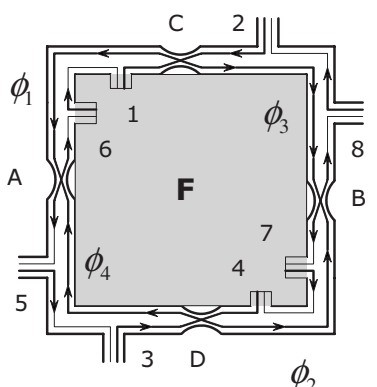

(b)
FIG. 1. A two-electron MZI. (a) Two-electron sources (a and b) and two detectors (c and d) connected by chiral edges. An AB flux is threading the interferometer. The sensitivity of the current cross correlation in the flux is the result of the indistinguishability of electrons emitted from a and b. Schematically, $\left\langle I_{c} I_{d}\right\rangle \propto\left|A_{b \rightarrow d} A_{a \rightarrow c}+A_{b \rightarrow c} A_{a \rightarrow d}\right|^{2}$, where $A_{b \rightarrow d}$ is the amplitude for transmission for $b$ to $d$. For example, the combination $A_{b \rightarrow d^{d}} A_{a \rightarrow c} A_{b \rightarrow c}^{*} A_{a \rightarrow d}^{*}=A_{b \rightarrow d} A_{d \rightarrow a} A_{a \rightarrow c} A_{c \rightarrow b}$ is phase sensitive. (b) A realistic setup with edge states II (Ref. 2) discussed in the text. 
proach, one encounters certain technical difficulties related to the current conservation, and we show how they can be fixed by introducing a number of constraints for scattering matrices. In principle, the derivation of these constraints can come from the full scattering theory with interactions (not available yet), however, we believe that our considerations are general enough and hold for an arbitrary scattering setup. In particular, we note that the present manifestation of MZI requires operating in the presence of a strong magnetic field. This means that the electrons are likely to be spin polarized, and seemingly, the physics discussed here has no practical implication. We, nevertheless, maintain that the mechanism of entangled-related suppression of two-particle interferometry is quite general, and the example discussed here of a spin-entangled electron pair is only a conceptually convenient example.

Below, we first derive general expressions for the current and noise of a generic multiterminal system, in which electrons in the same or different leads can be entangled. Let us consider a multiterminal system, with the leads emitting (possibly) entangled electrons. A two-electron state in such a system has the form

$$
\hat{F}_{\alpha \beta}^{\dagger}\left(E, E^{\prime}\right)|0\rangle=\sum_{\sigma, \sigma^{\prime}} g_{\sigma \sigma^{\prime}}^{* \alpha \beta} \hat{a}_{\alpha \sigma}^{\dagger}(E) \hat{a}_{\beta \sigma^{\prime}}^{\dagger}\left(E^{\prime}\right)|0\rangle,
$$

where the indices $\alpha$ and $\beta$ label the leads, $\sigma$ and $\sigma^{\prime}$ label the spin projections, and the coefficient $g$ is essentially the density matrix for the entangled electron state. ${ }^{10}$ For simplicity, we assume that each lead supports a single channel: The energies $E$ and $E^{\prime}$ describe the longitudinal motion. The generalization to the multichannel case is straightforward. The state is entangled if it cannot be constructed as a product of creation and annihilation operators acting on the vacuum. Below, we use as a reference state the state of electron pairs emitted by nonpolarized electrons,

$$
\left[\hat{a}_{\alpha \uparrow}^{\dagger}(E)+\hat{a}_{\alpha \downarrow}^{\dagger}(E)\right]\left[\hat{a}_{\beta \uparrow}^{\dagger}\left(E^{\prime}\right)+\hat{a}_{\beta \downarrow}^{\dagger}\left(E^{\prime}\right)\right]|0\rangle,
$$

to be referred as a full product state. For the full product state, the theory must retrieve the results of a single-electron scattering approach employed to describe transport.

\section{CURRENT AND NOISE}

The two-particle field operator depending on the coordinates, times, and spin projections of both particles is

$$
\begin{aligned}
\hat{\Psi}_{\sigma_{1} \sigma_{2}}\left(x_{1} t_{1} ; x_{2} t_{2}\right)= & \frac{1}{2 \pi \hbar v} \sum_{\beta \gamma} \int d E_{1} d E_{2} \phi_{\beta}\left(x_{1}, E_{1}\right) \phi_{\gamma}\left(x_{2}, E_{2}\right) \\
& \times \exp \left(-i E_{1} t_{1} / \hbar-i E_{2} t_{2} / \hbar\right) g_{\sigma_{2} \sigma_{1}}^{\gamma \beta} \hat{a}_{\beta \sigma_{1}} \hat{a}_{\gamma \sigma_{2}},
\end{aligned}
$$

where, for simplicity, we take the electron velocities in all leads equal to $v$, and $\phi_{\nu}(x)$ is the scattering state originating from the lead $\nu$. The asymptotic form for this function, for $x \in \alpha$, is

$$
\phi_{\nu}(x, E)=\delta_{\alpha \nu} e^{-i k(E) x}+s_{\alpha \nu}(E) e^{i k(E) x}
$$

where, as common in the scattering approach, the coordinate $x$ is counted along each lead (the origin is not important) and $\hat{s}$ is the scattering matrix (below, it will gain some more indices). The scattering states are orthogonal, in discrete notations $\int d x \phi_{\mu}^{*}(x, E) \phi_{\nu}\left(x, E^{\prime}\right)=L \delta_{\mu \nu} \delta_{E E^{\prime}}$, with $L$ being the length of the lead (it drops out of the final expressions). ${ }^{11}$

Now, we produce the one-particle current operator in the lead $\alpha$. It consists of the current operators produced by the "first" $\left(\hat{I}_{1}\right)$ and the "second" $\left(\hat{I}_{2}\right)$ particles. We write

$$
\begin{aligned}
\hat{I}_{\alpha 1}\left(x_{1}, t_{1}\right)= & -\frac{i e \hbar}{2 m} \int d x_{2} \sum_{\sigma_{1} \sigma_{2}} \\
& \times\left\langle\hat{\Psi}_{\sigma_{1} \sigma_{2}}^{\dagger}\left(x_{1}, t_{1} ; x_{2}, t_{2}\right) \frac{\partial}{\partial x_{1}} \hat{\Psi}_{\sigma_{1} \sigma_{2}}\left(x_{1}, t_{1} ; x_{2}, t_{2}\right)\right\rangle_{2} \\
& + \text { H.c. } \quad \text { (wrong), }
\end{aligned}
$$

where $\langle\ldots\rangle_{2}$ means quantum-mechanical averaging over the state of the second particle. Using ${ }^{12}$

$$
\left\langle\hat{a}_{\lambda \sigma}^{\dagger}(E) \hat{a}_{\nu \sigma^{\prime}}\left(E^{\prime}\right)\right\rangle=\delta_{\lambda \nu} \delta_{\sigma \sigma^{\prime}} \delta\left(E-E^{\prime}\right) f\left(E-\mu_{\lambda}\right),
$$

where $f$ is the equilibrium distribution function and $\mu_{\lambda}$ is the corresponding chemical potential, we obtain for the singleparticle current operator,

$$
\begin{aligned}
\hat{I}_{\alpha 1}(x, t)= & -\frac{e}{2 \pi \hbar} \frac{L}{2 \pi \hbar v} \int d E_{1} d E_{2} e^{i\left(E_{1}-E_{2}\right) t / \hbar} \sum_{\beta \gamma \delta \sigma_{1} \sigma_{2}} \sum \\
& \times A_{\beta \delta}^{\alpha}\left(E_{1}, E_{2}\right) N_{\gamma \sigma_{2}} g_{\sigma_{1} \sigma_{2}}^{*} g_{\sigma_{1} \sigma_{2}}^{\delta \gamma} \hat{a}_{\beta \sigma_{1}}^{\dagger}\left(E_{1}\right) \hat{a}_{\delta \sigma_{1}}\left(E_{2}\right) N_{\gamma \sigma_{2}} \\
& \text { (still wrong), }
\end{aligned}
$$

where we have introduced the following combination of the scattering matrices, ${ }^{13}$

$$
\begin{aligned}
A_{\beta \delta}^{\alpha}\left(E_{1}, E_{2}\right) \equiv & \delta_{\alpha \beta} \delta_{\alpha \delta} e^{i\left[k\left(E_{1}\right)-k\left(E_{2}\right)\right] x} \\
& -s_{\alpha \beta}^{*}\left(E_{1}\right) s_{\alpha \delta}\left(E_{2}\right) e^{-i\left[k\left(E_{1}\right)-k\left(E_{2}\right)\right] x}
\end{aligned}
$$

and the quantity $N_{\alpha \sigma} \equiv \int d E f_{\alpha \sigma}(E) d E$, which is proportional to the "number of particles" with the spin $\sigma$ emitted by the lead $\gamma$.

The expression for $\hat{I}_{\alpha 1}$ is obviously wrong: It yields the average current proportional to the number of pairs of particles and not to the difference of the number of particles emitted by each lead. This is because our expression for the current operator is an overcounting: Each pair of the particles is counted twice. Thus, we need to add a normalization constant in the current operator. This normalization constant is the number of pairs which a particle incident from the lead $\gamma$ with the spin $\sigma_{2}$ forms, divided by the number of such particles. Note that this constant must depend on $\gamma$ and $\sigma_{2}$. Let us now calculate it, 


$$
\begin{aligned}
\text { No. of pairs }= & \int d x_{1} d x_{2} d E_{1} d E_{2} d E_{3} d E_{4} \sum_{\beta \sigma_{1}} \frac{1}{(2 \pi \hbar v)^{2}} \\
& \times \phi_{\beta}^{*}\left(x_{1}, t_{1}, E_{1}\right) \phi_{\gamma}^{*}\left(x_{2}, t_{2}, E_{2}\right) \phi_{\gamma}\left(x_{2}, t_{2}, E_{3}\right) \\
& \times \phi_{\beta}\left(x_{1}, t_{1}, E_{4}\right)\left|g_{\sigma_{1} \sigma_{2}}^{\beta \gamma}\right|^{2} \\
& \times\left\langle a_{\beta \sigma_{1}}^{\dagger}\left(E_{1}\right) a_{\gamma \sigma_{2}}^{\dagger}\left(E_{2}\right) a_{\gamma \sigma_{2}}\left(E_{3}\right) a_{\beta \sigma_{1}}\left(E_{4}\right)\right\rangle \\
= & \left(\frac{L}{2 \pi \hbar v}\right)^{2} \sum_{\beta \sigma_{1}}\left|g_{\sigma_{1} \sigma_{2}}^{\beta \gamma}\right|^{2} N_{\beta \sigma_{1}} N_{\gamma \sigma_{2}},
\end{aligned}
$$

and No. of particles $=(L / 2 \pi \hbar v) N_{\gamma \sigma_{2}}$. Thus, including the normalization constant, we find for the current operator of the first particle,

$$
\begin{aligned}
\hat{I}_{\alpha 1}(x, t)= & -\frac{e}{2 \pi \hbar} \int d E_{1} d E_{2} e^{i\left(E_{1}-E_{2}\right) t / \hbar} \sum_{\beta \gamma \delta \sigma_{1} \sigma_{2}} \sum_{\nu \sigma} \frac{g_{\sigma_{1} \sigma_{2}}^{* \beta \gamma} g_{\sigma_{1} \sigma_{2}}^{\delta \gamma} N_{\gamma \sigma_{2}}}{\sum_{\nu \sigma_{2}}\left|g^{\nu \gamma}\right|^{2} N_{\nu \sigma}} \\
& \times A_{\beta \delta}^{\alpha}\left(E_{1}, E_{2}\right) \hat{a}_{\beta \sigma_{1}}^{\dagger}\left(E_{1}\right) \hat{a}_{\delta \sigma_{1}}\left(E_{2}\right) N_{\gamma \sigma_{2}},
\end{aligned}
$$

and an identical expression for the current operator of the second particle.

Next, we use Eq. (4) to derive the average current,

$$
\begin{aligned}
\left\langle I_{\alpha}\right\rangle= & \left\langle I_{\alpha 1}\right\rangle+\left\langle I_{\alpha 2}\right\rangle= \\
& -\frac{e}{2 \pi \hbar} \sum_{\gamma \sigma_{2}} \frac{\sum_{\beta \sigma_{1}} A_{\beta \beta}^{\alpha}\left(\gamma \sigma_{2}\right)\left|g_{\sigma_{1} \sigma_{2}}^{\beta \gamma}\right|^{2} N_{\beta \sigma_{1}} N_{\gamma \sigma_{2}}}{\sum_{\beta \sigma_{1}}\left|g_{\sigma_{1} \sigma_{2}}^{\beta \gamma}\right|^{2} N_{\beta \sigma_{1}}},
\end{aligned}
$$

where we assumed that the scattering matrices are energy independent, and we added to them two additional indices, as is explained below.

Equation (4) also yields current noise. Averaging the product of four creation and annihilation operators, ${ }^{13}$

$$
\begin{aligned}
\left\langle\hat{a}_{\beta \sigma}^{\dagger}\right. & \left.\left(E_{1}\right) \hat{a}_{\beta^{\prime} \sigma^{\prime}}\left(E_{1}^{\prime}\right) \hat{a}_{\delta \sigma^{\prime}}^{\dagger}\left(E_{2}\right) \hat{a}_{\delta^{\prime} \sigma^{\prime}}\left(E_{2}^{\prime}\right)\right\rangle-\left\langle\hat{a}_{\beta \sigma}^{\dagger}\left(E_{1}\right) \hat{a}_{\beta^{\prime} \sigma^{\prime}}\left(E_{1}^{\prime}\right)\right\rangle \\
\times & \left\langle\hat{a}_{\delta \sigma}^{\dagger}\left(E_{2}\right) \hat{a}_{\delta^{\prime} \sigma^{\prime}}\left(E_{2}^{\prime}\right)\right\rangle \\
= & \delta_{\beta \delta^{\prime}} \delta_{\beta^{\prime} \delta} \delta_{\sigma \sigma^{\prime}} \delta\left(E_{1}-E_{2}^{\prime}\right) \delta\left(E_{1}^{\prime}-E_{2}\right) f_{\beta \sigma}\left(E_{1}\right) \\
& \times\left[1-f_{\beta^{\prime} \sigma}\left(E_{2}\right)\right],
\end{aligned}
$$

we obtain

$$
\begin{aligned}
& S_{\alpha \alpha^{\prime}}=\frac{e^{2}}{2 \pi \hbar} \sum_{\beta, \beta^{\prime}, \gamma, \gamma^{\prime}} \sum_{\sigma_{1}, \sigma_{2}, \sigma_{2}^{\prime}} \frac{N_{\gamma \sigma_{2}}}{\sum_{\beta \sigma_{1}}\left|g_{\sigma_{1} \sigma_{2}}^{\beta \gamma}\right|^{2} N_{\beta \sigma_{1}}} \frac{N_{\gamma^{\prime} \sigma_{2}^{\prime}}}{\sum\left|g_{\sigma_{1} \sigma_{2}}^{\beta \gamma^{\prime}}\right|^{2} N_{\beta \sigma_{1}}} \\
& \times \operatorname{Re}\left[g_{\sigma_{1} \sigma_{2}}^{* \beta \gamma} g_{\sigma_{1} \sigma_{2}}^{\beta^{\prime} \gamma} g_{\sigma_{1} \sigma_{2}^{\prime}}^{* \beta^{\prime} \gamma^{\prime}} g_{\sigma_{1} \sigma_{2}^{\prime}}^{\beta \gamma^{\prime}}\right] A_{\beta \beta^{\prime}}^{\alpha}\left(\gamma, \sigma_{2}\right) A_{\beta^{\prime} \beta}^{\alpha^{\prime}}\left(\gamma^{\prime}, \sigma_{2}^{\prime}\right) \\
& \times \int d E f_{\beta \sigma_{1}}(E)\left[1-f_{\beta^{\prime} \sigma_{1}}(E)\right]+\left(\alpha \leftrightarrow \alpha^{\prime}\right) .
\end{aligned}
$$

Equations (5) and (6) generalize standard expressions for the multiterminal current and noise to the case of entangled electrons. It is easy to check that if electrons are not entangled and are in the product state, the coefficients $g$ are equal to one for all values of the arguments, and these expressions reduce to the standard one-particle formulas. ${ }^{13}$

The problem with Eqs. (5) and (6) is that currents are not automatically conserved, $\Sigma_{\alpha} I_{\alpha} \neq 0$. Also, if all leads have equal chemical potentials, nonvanishing currents can be still generated according to Eq. (5). Usually, requirements of current conservation and gauge invariance (currents are unchanged if the chemical potentials of all reservoirs are shifted simultaneously) are guaranteed by the unitarity of the scattering matrix. In our case, they are satisfied automatically provided that we choose the scattering matrix to obey

$$
\begin{gathered}
\sum_{\mu} s_{\mu \nu}^{*}\left(\gamma, \sigma_{2}\right) s_{\mu \nu^{\prime}}\left(\gamma^{\prime}, \sigma_{2}^{\prime}\right)=\delta_{\nu \nu^{\prime}}, \\
\sum_{\nu} g_{\sigma \sigma_{2}}^{* \nu \gamma} g_{\sigma \sigma_{2}^{\prime}}^{\nu \gamma^{\prime}} s_{\mu \nu}^{*}\left(\gamma, \sigma_{2}\right) s_{\mu^{\prime} \nu}\left(\gamma^{\prime}, \sigma_{2}^{\prime}\right)=\delta_{\mu \mu^{\prime}} g_{\sigma \sigma_{2}}^{*} g_{\sigma \sigma_{2}^{\prime}}^{\mu \gamma^{\prime}}
\end{gathered}
$$

\section{MACH-ZEHNDER INTERFEROMETER AND VISIBILITY}

Next, we specialize to the eight-terminal setup with edge states suggested by SSB. ${ }^{2}$ We will always bias sources 2 and 3 with the same voltage $V$ and measure the average current through lead 5 and the cross correlation between currents at 5 and 8. We are interested in the linear in $V$ effects. Assuming that $\mathrm{eV}$ is much smaller that the Fermi energy in the leads, we can, in the leading order, take all quantities $N_{\beta \sigma}$ to be the same (voltage independent). One has then

$$
\left\langle I_{5}\right\rangle=-\frac{e^{2} V}{2 \pi \hbar} \sum_{\gamma \sigma_{2}} \frac{1}{\sum_{\beta \sigma_{1}}\left|g_{\sigma_{1} \sigma_{2}}^{\beta \gamma}\right|^{2}} \sum_{\beta=2,3} \sum_{\sigma_{1}}\left|s_{5 \beta}\left(\gamma, \sigma_{2}\right)\right|^{2}
$$

and

$$
\begin{aligned}
S_{58}= & -\frac{e^{3}|V|}{\pi \hbar} \sum_{\gamma \gamma^{\prime}} \sum_{\sigma_{1} \sigma_{2} \sigma_{2}^{\prime}} \frac{1}{\sum_{\beta \sigma_{1}}\left|g_{\sigma_{1} \sigma_{2}}^{\beta \gamma}\right|^{2}} \frac{1}{\sum_{\beta \sigma_{1}}\left|g_{\sigma_{1} \sigma_{2}^{\prime}}^{\beta \gamma^{\prime}}\right|^{2}} \\
& \times\left|\sum_{\beta=2,3} g_{\sigma_{1} \sigma_{2}}^{* \beta \gamma} g_{\sigma_{1} \sigma_{2}^{\prime}}^{\beta \gamma^{\prime}} s_{5 \beta}^{*}\left(\gamma, \sigma_{2}\right) s_{8 \beta}\left(\gamma^{\prime}, \sigma_{2}^{\prime}\right)\right|^{2},
\end{aligned}
$$

where we have used the "unitarity" conditions [Eq. (7)].

Now, we apply these expressions to the different states emitted by the reservoirs. The idea is to investigate under which conditions the electrons emitted from sources 2 and 3 are "painted," at least partially "red" and "blue," respectively, hence suppression of the flux sensitive (coherent) term of the correlation.

(i) Full product state, $g_{\sigma \sigma^{\prime}}^{\beta \gamma}=1$. This is the case considered by SSB. Choosing $s_{52}=t_{A} t_{C} e^{i \phi_{1}}, s_{53}=r_{A} r_{D} e^{i \phi_{4}}, s_{82}=r_{B} r_{C} e^{i \phi_{3}}$, and $s_{83}=t_{B} t_{D} e^{i \phi_{2}}$, where $t$ and $r$ are the transmission and reflection amplitudes of the corresponding quantum point contacts (Fig. 1), we obtain 


$$
\left\langle I_{5}\right\rangle=-\frac{e^{2} V}{2 \pi \hbar}\left(\left|s_{52}\right|^{2}+\left|s_{53}\right|^{2}\right)=-\frac{e^{2} V}{2 \pi \hbar}\left(T_{A} T_{C}+R_{A} R_{D}\right),
$$

where $T$ and $R$ are corresponding transmission and reflection probabilities, and

$$
\begin{aligned}
S_{58} & =-\frac{2 e^{3}|V|}{\pi \hbar}\left|s_{52}^{*} s_{82}+s_{53}^{*} s_{83}\right|^{2} \\
& =-\frac{2 e^{3}|V|}{\pi \hbar}\left|\sqrt{T_{A} T_{C} R_{B} R_{C}} e^{i \theta}+\sqrt{T_{B} T_{D} R_{A} R_{D}}\right|^{2},
\end{aligned}
$$

where $\theta$ is a linear combination of the phases of transmission and reflection amplitudes and the Aharonov-Bohm phase. In accordance with SSB, the average current is not sensitive to $\mathrm{AB}$ effect, since not a single-electron trajectory encircles the magnetic flux. By contrast, noise is a two-particle phenomenon, and the setup is designed in such a way that the cross correlations are sensitive to the $\mathrm{AB}$ flux. The visibility of the noise oscillations is

$$
v=\frac{2 \sqrt{T_{A} T_{B} T_{C} T_{D} R_{A} R_{B} R_{C} R_{D}}}{T_{A} T_{C} R_{B} R_{C}+T_{B} T_{D} R_{A} R_{D}},
$$

and it becomes unity (ideal oscillations) provided the setup is symmetric: all transmission and reflection probabilities equal to $1 / 2$. This is the result of SSB.

(ii) One source is spin polarized. Imagine that states are still not entangled but source 2 is spin polarized: It only emits and absorbs spin-up electrons. The coefficients $g$ are all equal to one, with the exception of $g_{\downarrow \sigma_{2}}^{2 \gamma}=g_{\sigma_{1} \downarrow}^{\beta 2}=0$. We need now to choose the scattering matrices in such a way so that they obey the unitarity condition (7). This is easily done intuitively. For spin-up electrons, the constraints are the same as for the full product state, and thus, the scattering for spin-up electrons is described by the matrix $s$. The scattering matrix for spin-down electrons, which we denote as $\widetilde{s}$, is constrained by the condition

$$
\sum_{\nu \neq 2} \widetilde{s}_{\mu \nu}^{*} \tilde{s}_{\mu^{\prime} \nu}=\delta_{\mu \mu^{\prime}}, \quad \widetilde{s}_{\mu 2}=0 .
$$

We obtain the average current,

$$
\left\langle I_{5}\right\rangle=-\frac{e^{2} V}{2 \pi \hbar}\left(\left|s_{52}\right|^{2}+\left|s_{53}\right|^{2}+\left|\widetilde{s}_{53}\right|^{2}\right),
$$

and current noise,

$$
S_{58}=-\frac{e^{3}|V|}{\pi \hbar}\left(\left|s_{52}^{*} s_{82}+s_{53}^{*} s_{83}\right|^{2}+\left|\widetilde{s}_{53}^{*} \widetilde{s}_{83}\right|^{2}\right) .
$$

The first term in the brackets, similarly to Eq. (11), contains both phase-insensitive and $\mathrm{AB}$ terms. The second term is insensitive to the $\mathrm{AB}$ phase. Thus, the total visibility in the noise is reduced by the presence of this second term. The visibility depends on the choice of the scattering matrices $\widetilde{s}$, in particular, if the setup is symmetric, and $\widetilde{s}_{53}=0$ (unrealistic case); the oscillations are still ideal $(v=1)$. This is because, in this case, a spin-down electron, originating from 3 , has to go to 8 with certainty, and thus, it does not change the cur- rent noise. On the other hand, the natural choice would be $\left|s_{52}\right|=\left|s_{53}\right|=\left|\widetilde{s}_{52}\right|$. In this case, which we call optimal, the visibility equals $v=2 / 3$.

In the same way, we can treat a setup where both sources, 2 and 3 , are polarized. If they are polarized in the same direction (spin up), the visibility is the same as for full product states (both current and noise are reduced by the factor of 2 since now, only spin-up states contribute). Provided one source is spin-up polarized and the other one is spin down, the visibility vanishes: There are no $\mathrm{AB}$ oscillations in this case, since one can say with certainty from what source each electron has originated.

(iii) Entangled electrons from one source. Pairs in triplet $s_{z}=0$ state are emitted from lead 2 ,

$$
|2 \uparrow\rangle|2 \downarrow\rangle+|2 \downarrow\rangle|2 \uparrow\rangle,
$$

all other leads are in the full product state. This means that all the coefficients $g$ are equal to one except for $g_{\uparrow \uparrow}^{22}=g_{\downarrow \downarrow}^{22}=0$. Now, we are obliged to choose scattering matrices which depend on the second electron in the pair-the "spouse"-otherwise the unitarity (7) conditions cannot be fulfilled. We choose

$$
s_{\alpha \nu, \sigma}\left(\gamma, \sigma_{2}\right)=\left\{\begin{array}{lrl}
s_{\alpha \nu}, & \gamma \neq 2 \text { or } \gamma=2, & \sigma \neq \sigma_{2} \\
\tilde{s}_{\alpha \nu}, & \gamma=2, & \sigma=\sigma_{2} .
\end{array}\right.
$$

Note that this choice (affecting the visibility) is arbitrary and definitely not unique. Our matrices now, in addition to what we have already seen, obey

$$
\sum_{\nu \neq 2} \widetilde{s}_{\mu \nu}^{*} s_{\mu^{\prime} \nu}=\sum_{\nu \neq 2} s_{\mu \nu}^{*} \widetilde{s}_{\mu^{\prime} \nu}=\delta_{\mu \mu^{\prime}} .
$$

We can again calculate the average current,

$$
\left\langle I_{5}\right\rangle=-\frac{e^{2} V}{2 \pi \hbar}\left\{\left(\frac{7}{8}+\frac{1}{15}\right)\left(\left|s_{52}\right|^{2}+\left|s_{53}\right|^{2}\right)+\frac{1}{15}\left|\widetilde{s}_{53}\right|^{2}\right\},
$$

and the current noise,

$$
\begin{aligned}
S_{58}= & -\frac{2 e^{3}|V|}{\pi \hbar}\left\{\left(\frac{14^{2}}{16^{2}}+2 \frac{14}{15 \times 16}+\frac{1}{15^{2}}\right)\left|s_{52}^{*} s_{82}+s_{53}^{*} s_{83}\right|^{2}\right. \\
& \left.+\left(\frac{14}{15 \times 16}+\frac{1}{15^{2}}\right)\left(\left|\tilde{s}_{53}^{*} s_{83}\right|^{2}+\left|s_{53}^{*} \widetilde{s}_{83}\right|^{2}\right)+\frac{1}{15^{2}}\left|\widetilde{s}_{53}^{*} \widetilde{s}_{83}\right|^{2}\right\} .
\end{aligned}
$$

Again, phase-dependent contributions are only found in the first term in the braces. All other terms are phase insensitive and thus reduce the visibility. In particular, with the same optimal choice of scattering matrices, $\left|s_{52}\right|=\left|s_{53}\right|=\left|\widetilde{s}_{52}\right|$, the visibility is reduced to 0.93 .

(iv) Pairs in singlet state are emitted from lead 2,

$$
|2 \uparrow\rangle|2 \downarrow\rangle-|2 \downarrow\rangle|2 \uparrow\rangle .
$$

All other leads are in the full product state. This means that all the coefficients $g$ are equal to one except for $g_{\uparrow \uparrow}^{22}=g_{\downarrow \downarrow}^{22}=0$ and $g_{\downarrow \uparrow}^{22}=-1$. We choose the scattering matrices in the following way (this choice is again arbitrary): 
TABLE I. Reduction of the visibility for different scenarios and, otherwise, optimal conditions.

\begin{tabular}{lc}
\hline \hline Scenario & Optimal visibility \\
\hline 2, 3: product states & 1 \\
2: product, not polarized; 3: polarized $\uparrow$ & $2 / 3$ \\
2, 3: polarized $\uparrow$ & 1 \\
2: polarized $\uparrow ;$ 3: polarized $\downarrow$ & 0 \\
2: entangled, singlet; 3: product & 0.93 \\
2: entanglet, triplet; 3: product & 0.93 \\
\hline \hline
\end{tabular}

$$
s_{\alpha \nu, \sigma}\left(\gamma, \sigma_{2}\right)=\left\{\begin{array}{lll}
s_{\alpha \nu}, & \gamma \neq 2 \text { or } \gamma=2, & \left(\sigma \sigma_{2}\right)=(\uparrow \downarrow) \\
\widetilde{s}_{\alpha \nu}, & \gamma=2, & \sigma=\sigma_{2} \\
\bar{s}_{\alpha \nu}, & \gamma=2, & \left(\sigma \sigma_{2}\right)=(\downarrow \uparrow)
\end{array} .\right.
$$

The matrix $\bar{s}$ must then obey the following constraints:

$$
\begin{aligned}
\sum_{\nu} \bar{s}_{\mu \nu}^{*} \bar{s}_{\mu^{\prime} \nu} & =\sum_{\nu} s_{\mu \nu^{*}}^{*} \bar{s}_{\mu^{\prime} \nu}=\sum_{\nu} \bar{s}_{\mu \nu}^{*} s_{\mu^{\prime} \nu}=\sum_{\nu \neq 2} \bar{s}_{\mu \nu}^{*} \tilde{s}_{\mu^{\prime} \nu} \\
& =\sum_{\nu \neq 2} \widetilde{s}_{\mu \nu^{*}}^{*} \bar{s}_{\mu^{\prime} \nu}=\delta_{\mu \mu^{\prime}} .
\end{aligned}
$$

With this choice of scattering matrices, we obtain the same current and current noise (and consequently, the same visibility) as for the triplet $s_{z}=0$ state. We should recall, however, that this conclusion depends on the choice of scattering matrices [Eq. (17)]: for instance, on the fact that $s_{\alpha \nu, \sigma}(2, \sigma)$ is the same for single and triplet entangled states.

\section{CONCLUSIONS}

In summary, we have defined and analyzed various scenarios for which single-particle amplitudes maintain their coherence (as would be manifest in a Mach-Zehnder interferometry measurement), yet (partial) distinguishability of electrons emitted from different sources suppresses the flux sensitivity of the two-particle cross-correlation function (Table I). Our analysis demonstrates how two-particle entanglement can give rise to a behavior akin to a "dephasing" of a two-particle interferometry.

\section{ACKNOWLEDGMENTS}

We acknowledge useful discussions with M. Büttiker, M. Heiblum, I. Neder, and P. Samuelsson. This work was supported by the ISF of the Israel Academy of Sciences, by the US-Israel BSF, by the Minerva Einstein Center (BMBF), and by the Transnational Access Program (RITA-CT-2003506095) at the Weizmann Institute of Science.
${ }^{1}$ Y. Ji, Y. Chung, D. Sprinzak, M. Heiblum, D. Mahalu, and H. Shtrikman, Nature (London) 422, 415 (2003).

${ }^{2}$ P. Samuelsson, E. V. Sukhorukov, and M. Büttiker, Phys. Rev. Lett. 92, 026805 (2004); see also V. S.-W. Chung, P. Samuelsson, and M. Büttiker, Phys. Rev. B 72, 125320 (2005).

${ }^{3}$ Still, dephasing might be responsible for the suppression of visibility in the experiment (Ref. 1), see F. Marquardt and C. Bruder, Phys. Rev. Lett. 92, 056805 (2004); I. Neder and F. Marquardt, Nev. J. Phys. 9, 112 (2007); J. T. Chalker, Y. Gefen, and M. Y. Veillette, Phys. Rev. B 76, 085320 (2007).

${ }^{4}$ C. W. J. Beenakker, Proceedings of the International School Physics Enrico Fermi (IOS, Amsterdam, 2006), Vol. 162; G. Burkard, J. Phys.: Condens. Matter 19, 233202 (2007).

${ }^{5}$ A. V. Lebedev, G. Blatter, C. W. J. Beenakker, and G. B. Lesovik, Phys. Rev. B 69, 235312 (2004); A. V. Lebedev, G. B. Lesovik, and G. Blatter, ibid. 71, 045306 (2005).

${ }^{6}$ A. Di Lorenzo and Yu. V. Nazarov, Phys. Rev. Lett. 94, 210601 (2005)

${ }^{7}$ C. W. J. Beenakker, C. Emary, M. Kindermann, and J. L. van Velsen, Phys. Rev. Lett. 91, 147901 (2003).
${ }^{8}$ D. E. Feldman, Y. Gefen, A. Kitaev, K. T. Law, and A. Stern, Phys. Rev. B 76, 085333 (2007).

${ }^{9}$ S. Das Sarma, M. Freedman, C. Nayak, S. H. Simon, and A. Stern, arXiv:0707.1889 (unpublished).

${ }^{10} \mathrm{We}$ take the coefficients $g$, to be energy independent. A different choice, for instance $g \propto \delta\left(E-E^{\prime}\right)$, may yield different results, G. Burkard, D. Loss, and E. V. Sukhorukov, Phys. Rev. B 61, R16303 (2000).

${ }^{11}$ It will be more natural and convenient to use continuous notations for the energy $\int d x \phi_{\mu}^{*}(x, E) \phi_{\nu}\left(x, E^{\prime}\right)=2 \pi \hbar v \delta_{\mu \nu} \delta\left(E-E^{\prime}\right)$. In particular, one can also write the "square" of the delta function in continuous notations $\delta^{2}\left(E-E^{\prime}\right)=(L / 2 \pi v) \delta\left(E-E^{\prime}\right)$.

${ }^{12}$ Note that as a consequence of our choice of the field operator [Eq. (2)], the rules for the averaging imply that entangled electron pairs are characterized by the Fermi distribution function. Whereas such a choice is plausible, the actual distribution function may depend on the mechanism of entanglement. We thank M. Büttiker for useful comments to this point.

${ }^{13}$ M. Büttiker, Phys. Rev. B 46, 12485 (1992); see also Ya. M. Blanter and M. Büttiker, Phys. Rep. 336, 1 (2000). 